\title{
Sciendo
}

\author{
BULGARIAN ACADEMY OF SCIENCES
}

CYBERNETICS AND INFORMATION TECHNOLOGIES • Volume 20, No 4

Sofia $\bullet 2020 \quad$ Print ISSN: 1311-9702; Online ISSN: 1314-4081

DOI: $10.2478 /$ cait-2020-0053

\section{Predictive Analysis of Dengue Outbreak Based on an Improved Salp Swarm Algorithm}

\author{
Zuriani Mustaffa ${ }^{1}$, Mohd Herwan Sulaiman ${ }^{2}$, Khairunnisa Amalina \\ Mohd Rosli ${ }^{1}$, Mohamad Farhan Mohamad Mohsin ${ }^{3}$, Yuhanis Yusof \\ ${ }^{1}$ Faculty of Computing, Universiti Malaysia Pahang, Pekan, Malaysia \\ ${ }^{2}$ Faculty of Electrical \& Electronics Engineering Technology, Universiti Malaysia Pahang, Pekan, \\ Malaysia \\ ${ }^{3}$ School of Computing, Universiti Utara Malaysia \\ E-mails:zuriani@ump.edu.my_mherwan@ieee.org_khairunnisaamalina@gmail.com \\ farhan@uum.edu.myyuhanis@uum.edu.my
}

\begin{abstract}
The purpose of this study is to enhance the exploration capability of conventional Salp Swarm Algorithm (SSA) with the inducing of Levy Flight. With such modification, it will assist the SSA from trapping in local optimum. The proposed approach, which is later known as an improved SSA (iSSA) is employed in monthly dengue outbreak prediction. For that matter, monthly dataset of rainfall, humidity, temperature and number of dengue cases were employed, which render prediction information. The efficiency of the proposed algorithm is evaluated using Root Mean Square Error (RMSE), and compared against the conventional SSA and Ant Colony Optimization (ACO). The obtained results suggested that the iSSA was not only able to produce lower RMSE, but also capable to converge faster at lower rate as well.
\end{abstract}

Keywords: Dengue outbreak prediction, meta-heuristic, optimization, predictive analysis, Salp Swarm Algorithm, swarm intelligence.

\section{Introduction}

Prediction issue is one of the fields that has been successfully addressed with the application of meta-heuristic based approach $[1,2]$. The importance of prediction is inevitable, regardless of the problem at hand, and this includes finance [3-5], cloud computing [6,7], electrical engineering [8], oil and gas engineering [9], and medical $[10,11]$ to name a few. By having accurate prediction, the obtained future values could assist in preparing proper precaution steps and prevention by the interested parties. Consequently, negative impact for certain problem such as loss of profit, excessive load, or increase in infectious disease cases which could lead to possibility of death can be avoided. 
In infectious disease, a good number of researches can be found which critically discussed the efficiency of meta-heuristic based approach in dealing with the stated issue. In [10], Particle Swarm Optimization (PSO) was hybrid with Artificial Neural Network (ANN) for early detection of dengue disease. In the research, the employment of PSO was to optimize the values of ANN parameters. Later, with the obtained optimal values, the detection task was carried out by ANN. Findings of the study suggested that PSO-ANN was competent to produce better result compared to other identified approaches. Similarly in [12], a prediction model for dengue is presented based on hybrid Elman Levenberg Neural Network and Genetic Algorithm (GA). Realized on dengue data in Indonesia, the presented model demonstrated a positive performance for the case under study. Other than [12], the work in [13] also employed a hybrid model of GA and Neural Network based model. On the other hand, a study in [14] proposed four hybrid models of Grey Wolf Optimizer (GWO), Moth Flame Optimizer (MFO), Firefly Algorithm (FA) and Artificial Bee Colony (ABC) with Least Squares Support Vector Machines (LSSVM). The identified metaheuristic algorithms, which were individually hybrid with LSSVM showed competitive results.

Other than dengue cases, recently, the prediction for Coronavirus disease (Covid19) which has been declared as pandemic can be found in [11]. In the presented study, an improved Flower Pollination Algorithm (FPA) based on Salp Swarm Algorithm (SSA) is hybrid with a modified Adaptive Neuro-Fuzzy Inference System (ANFIS). The presented work, which was tested on Covid19 data in USA and China showed good performances.

Even though numerous studies have been published concerning infectious disease prediction, the need to establish a reliable and efficient prediction model always exists, and this includes prediction model for dengue outbreak cases. According to [15], until today, there is no specific treatment offered for dengue fever, in fact, severe dengue disease could lead to dengue hemorrhagic fever, which is much riskier than dengue fever. It is worth noting that dengue is considered as the most widely distributed and rapidly spreading mosquito borne viral disease in the world and the number of incidences for dengue are continuously increasing which caught attention from many parties, including academic community [16, 17]. With respect to that matter, this study proposes a dengue outbreak prediction using a relatively new meta-heurictic alorithm namely Salp Swarm Algorithm (SSA) [18]. SSA is a relatively new meta-heuristic algorithm which has been successfully applied in various areas [19-21]. Nonetheless, the conventional SSA exposes to local optimum $[22,23]$ and prone to premature convergence $[24,19]$. Such situation cannot be ignored since it would demote the prediction performance. Concerning that matter, this work introduces a modification to conventional SSA based on Levy Flight [25]. The modification takes place in exploration phase, which is belived to alleviate its demerits. Later, the proposed model, termed as iSSA is compared againts conventional SSA and Ant Colony Optimization (ACO) for monthly dengue outbreak prediction. Further discussion is provided in the next sections.

The rest of this paper is structured as follow: Sections 2 and 3 provide a description on SSA and Levy Flight respetively. Section 4 discusses the obtained 
result while the analysis if is gven in Secion 5. Finally, Section 6 concludes the performance of the proposed method and the results obtained.

\section{Salp Swarm Algorithm}

This section describes the nature of salps and mathematical model of SSA. Detail description of SSA can be found in [18].

\subsection{Salps in nature}

Salp Swarm Algorithm [18] is a relatively new SI based algorithm which was proposed in 2017. Like any other SI algorithms, SSA is inspired based on intelligent behaviours of animal/insects in nature, where in this case, it is referring to salps. In nature, salps belong to the family of Salpidae, which comes with transparent-barrel shaped physical. Study shows that salps possess similarity with jelly fish in terms of their tissue structure and movement pattern. One of the unique behaviour of salps is their swarming behaviour which forming a salp chain. Fig. 1 shows an example of salp chain.

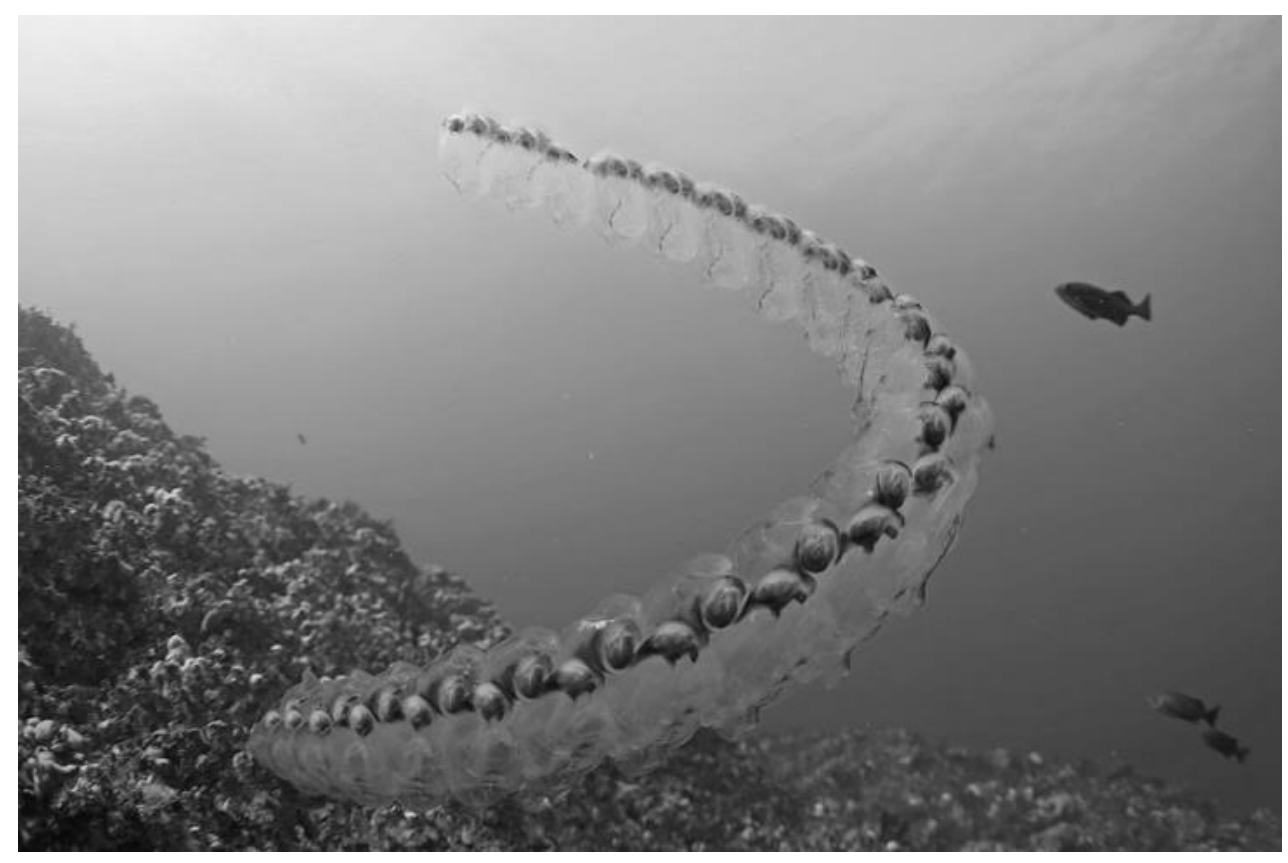

Fig. 1. An example of salp chain [26]

\subsection{Mathematical model of SSA}

During initialization stage, the salps population is splitted into two parts. The first part is incorporated of leader while the second part consists of followers. In the salp chain, the ones in the front line is designed as leader while the remaining are 
followers. The leader responsibles to guide the salp swarm while the followers follow each other (and leader directly or indirectly). It is worth noting that, in SSA, it only has one control parameter. The position of salps is defined in an $n$-dimensional space, where $n$ is referring to the number of variables for the problem at hands,

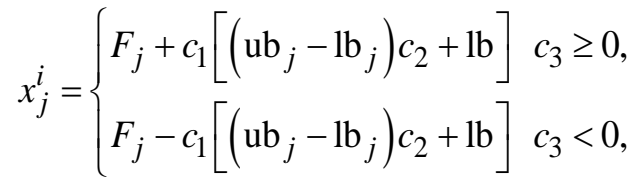

where:

$x_{j}^{i}$ is the position of the first salp, i.e., the leader,

$F_{j}$ is the food source position in the $j$-th dimension,

$\mathrm{ub}_{j}$ and $\mathrm{lb}_{j}$ are the upper bound and lower bound of $j$-th dimension, respectively, $c_{1}, c_{2}$ and $c_{3}$ are the random numbers.

As defined in (1), $x_{j}^{i}$ only updates its position with respect to the food source. Meanwhile, $c_{1}$ plays a vital role in balancing both exploitation and exploration processes. It is defined as

$$
c_{1}=2 e^{-\left(\frac{4 l}{L}\right)^{2}},
$$

where $l$ and $L$ indicate the current iteration and maximum number of iterations, respectively. Both $c_{2}$ and $c_{3}$ are random numbers uniformly generated in the interval of $[0,1]$. On the other hand, for followers, the position is updated as

$$
x_{j}^{i}=\frac{1}{2} a t^{2}+v_{0} t
$$

where:

$i \geq 2$,

$x_{j}^{i}$ is the position of $i$-th follower salp in $j$-th dimension,

$t$ is the time,

$v_{0}$ is the initial speed,

$a=\frac{v_{\text {final }}}{v_{0}}$, where $v=\frac{x-x_{0}}{t}$.

In optimization, the time is iteration. Due to that matter, the discrepancy between iteration is equal to 1 , and considering $v_{0}=0$, this is defined as

$$
x_{j}^{i}=\frac{1}{2}\left(x_{j}^{i}+x_{j}^{i-1}\right), i \geq 2,
$$

$x_{j}^{i}$ is the position of $i$-th follower salp in $j$-th dimension.

The simulation on salp chain can be simulated using Equations (1)-(4).

The pseudo cod of SSA is shown in Fig. 2. 


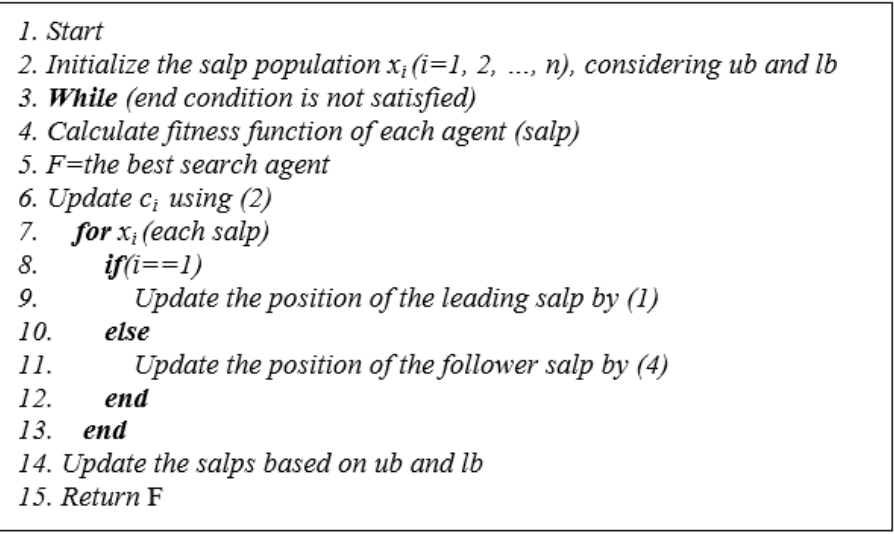

Fig. 2. SSA pseudo code

\section{Levy Flight}

The inducing of Levy Flight (LF) [25] to alleviate meta-heuristic algorithms is wellknown with its outstanding outcomes [27-29]. Mainly, the merits of LF depends on its variable namely $\beta$ where different values of $\beta$ will provide different random distribution. This unique characteristic leads to outstanding searching behaviour since it will lead to wider searching space, hence the incident of revisiting the similar location can be reduced. Fig. 3 shows pseudo code of LF that is implemented in this study.

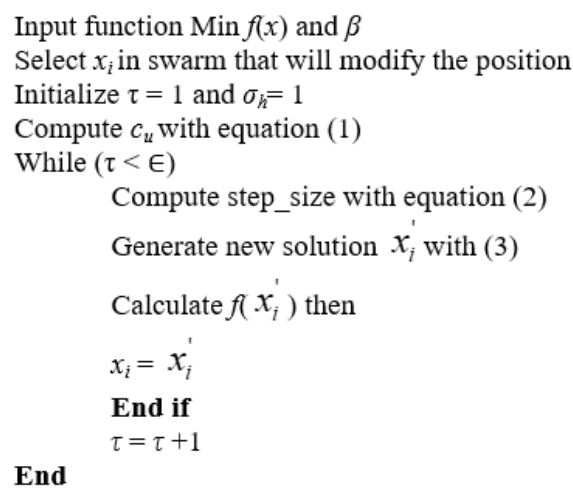

Fig. 3. Pseudo code of Levy Flight

$$
\sigma_{u}=\left\{\frac{\Gamma(1+\beta) \sin (\pi \beta / 2)}{\beta \Gamma[(1+\beta) / 2] 2^{\beta-1) / 2}}\right\},
$$




$$
x_{i j}^{\prime}(\tau+1)=x_{i j}(\tau)+\operatorname{step} \_\operatorname{size}(\tau) \times U(0,1) .
$$

The step size by using Levy distribution of search area is calculated as in (5). Factor of 0.01 is $L / 100$, where $L$ is typical length scale. In (6) where $x_{i j}$ is individual that will modify the position, $U(0,1)$ is a random number between $[0,1]$ range and step_size $(\tau) \times U(0,1)$ is the random walk from Levy distribution. In this study, the LF is applied to improve the exploration capability of SSA, which consequently lead to global optimum. This is the benefit from the remarkable performance of LF which is able to provide larger step length, i.e., better searching compared to Gaussian process [30].

\section{Methodology}

The implemented methodology incorporating data collection and pre-processing, experiment setup and evaluation. Fig. 4 shows the simplified form of the proposed methodology.

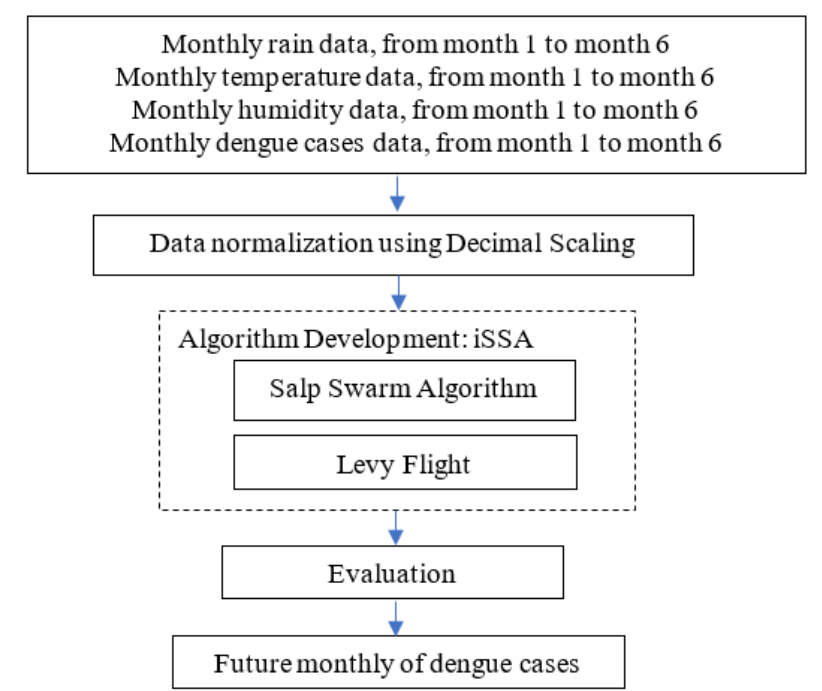

Fig. 4. Schematic of the proposed iSSA for dengue outbreak prediction

\subsection{Introducing the dataset}

The performance of iSSA was tested on dengue dataset, which is obtained from [31]. Besides the number of dengue incidence data, the data set also consist of three meteorological data which include cumulative rainfall $(\mathrm{mm})$, mean temperature readings $\left({ }^{\circ} \mathrm{C}\right)$, and mean relative humidity $(\%)$. These data sets are defined in monthly periodicity, from 2001-2013. From the downloaded data set, several missing values were identified. For that matter, the values were replaced with the average value of before and after the respective row. Table 1 shows the sample of original data recorded in 2013. 
Table 1. Sample of original data

\begin{tabular}{|l|c|c|c|c|}
\hline Month & Rain & Temperature & Humidity & Dengue cases \\
\hline January 2012 & 295.00 & 26.00 & 83.00 & 16 \\
\hline February 2012 & 388.00 & 26.00 & 83.00 & 39 \\
\hline March 2012 & 321.00 & 26.45 & 87.00 & 29 \\
\hline April 2012 & 247.00 & 26.68 & 87.00 & 44 \\
\hline May 2012 & 63.00 & 26.79 & 87.50 & 42 \\
\hline June 2012 & 4.00 & 26.84 & 87.75 & 36 \\
\hline July 2012 & 0.00 & 26.87 & 88.38 & 24 \\
\hline August 2012 & 33.50 & 26.89 & 87.69 & 16 \\
\hline September 2012 & 50.25 & 26.89 & 87.34 & 14 \\
\hline October 2012 & 67.00 & 26.90 & 88.17 & 25 \\
\hline November 2012 & 222.00 & 26.90 & 87.00 & 25 \\
\hline Dcember 2012 & 407.00 & 26.60 & 88.00 & 64 \\
\hline
\end{tabular}

\subsection{Data normalization}

Prior to training the inputs, all input and output values were normalized using Decimal Scaling,

(8)

$$
v^{\prime}=\left(v / 10^{j}\right)
$$

where:

$v^{\prime}$ is the new value of parameter,

$v$ is the old value,

$j$ is the smallest integer where $\operatorname{Max}\left(\left|v^{\prime}\right|\right)<1$. The sample of normalized data is tabulated in Table 2.

The normalized input for sample in Table 1 is as tabulated in Table 2.

Table 2. Sample of normalized data

\begin{tabular}{|l|c|c|c|c|}
\hline Month & Rain & Temperature & Humidity & Dengue cases \\
\hline January 2012 & 0.2950 & 0.2600 & 0.8300 & 0.0160 \\
\hline February 2012 & 0.3880 & 0.2600 & 0.8300 & 0.0390 \\
\hline March 2012 & 0.3210 & 0.2645 & 0.8700 & 0.0290 \\
\hline April 2012 & 0.2470 & 0.2668 & 0.8700 & 0.0440 \\
\hline May 2012 & 0.0630 & 0.2679 & 0.8750 & 0.0420 \\
\hline June 2012 & 0.0040 & 0.2684 & 0.8775 & 0.0360 \\
\hline July 2012 & 0.0000 & 0.2687 & 0.8838 & 0.0240 \\
\hline August 2012 & 0.0335 & 0.2689 & 0.8769 & 0.0160 \\
\hline September 2012 & 0.0502 & 0.2689 & 0.8734 & 0.0140 \\
\hline October 2012 & 0.0670 & 0.2690 & 0.8817 & 0.0250 \\
\hline November 2012 & 0.2220 & 0.2690 & 0.8700 & 0.0250 \\
\hline December 2012 & 0.4070 & 0.2660 & 0.8800 & 0.0640 \\
\hline
\end{tabular}

\subsection{Experiment structure}

This section consists of five sub-sections namely the arrangement of input and output in the iSSA prediction model, data division for training, validation and testing, mathematical model of iSSA, the iSSA prediction model and finally evaluation. 


\subsubsection{Input-output setup}

The input and output variables employed in this study are shown in Table 3 which incorporated of monthly data of rain, temperature, humidity and number of dengue cases. In addition, the derivation of the stated inputs is fed to the prediction model as well.

Table 3. Input and outputs

\begin{tabular}{|l|l|l|}
\hline Input & Variable & Output \\
\hline $\begin{array}{l}\text { Monthly rain data, from month 1 } \\
\text { to month 5 }\end{array}$ & $\begin{array}{l}\text { rain_m1, rain_m2, rain_m3, rain_m4, } \\
\text { rain_m5 }\end{array}$ & $\begin{array}{l}\text { Number of } \\
\text { dengue }\end{array}$ \\
\cline { 1 - 2 } $\begin{array}{l}\text { Monthly temperature data, } \\
\text { from month 1 to month 5 }\end{array}$ & $\begin{array}{l}\text { temp_m1, temp_m2, temp_m3, } \\
\text { cases, from } \\
\text { month 6 }\end{array}$ \\
$\begin{array}{lll}\text { Monthly humidity data, } \\
\text { from month 1 to month 5 }\end{array}$ & $\begin{array}{l}\text { hmdt_m1, temp_m5 } \\
\text { hmdt_m4, hmdt_m2, hmdt_m3, }\end{array}$ & $\begin{array}{l}\text { and onwards, } \\
\text { dcases_m6owd }\end{array}$ \\
\cline { 1 - 2 } $\begin{array}{l}\text { Monthly dengue cases number, from } \\
\text { month 1 to month 5 }\end{array}$ & $\begin{array}{l}\text { dcases_m1, dcases_m2, dcases_m3, } \\
\text { dcases_m4, dcases_m5 }\end{array}$ & \\
\hline
\end{tabular}

\subsubsection{Data division: Training, validation and testing}

From the employed data, the data is divided into three sets, as described in Table 4.

Table 4. Training, validation and testing

\begin{tabular}{|l|c|c|}
\hline Set & Data arrangement description & Proportion (\%) \\
\hline Training & $1-105$ & 70 \\
\hline Validation & $106-129$ & 15 \\
\hline Testing & $130-151$ & 15 \\
\hline
\end{tabular}

\subsubsection{Improved Salp Swarm Algorithm}

Similarly like any other meta-heuristic algorithms, SSA consists of exploitation and exploration phases, where both complement with each other in achieving global optimum. Therefore, it is vital for any meta heuristic algorithm to achieve a stability between both phases. In exploitation phase, the objective is to intensify the search in various areas of the increased search experience while in exploration the aim is to identify high quality solution through fragments of the search space.

In this study, an improvement to conventional SSA is introduced, where the objective is to enhance the exploration feature of SSA, hence better optimization performance can be obtained. For that matter, the advantage of Levy Distribution is benefited, which not only leads to better prediction performance but improves the convergence ability as well.

From (1), it is modified by inducing Levy Flight, as

$$
x_{j}^{i}= \begin{cases}F_{j}+c_{1}\left[\left(\mathrm{ub}_{j}-\mathrm{lb}_{j}\right) \mathrm{Lv}+\mathrm{lb}_{j}\right], & c_{3} \geq 0, \\ F_{j}-c_{1}\left[\left(\mathrm{ub}_{j}-\mathrm{lb}_{j}\right) \mathrm{Lv}+\mathrm{lb}_{j}\right], & c_{3}<0,\end{cases}
$$

where:

$x_{j}^{i}$ is the position of the first salp, i.e., the leader,

$F_{j}$ is the food source position in the $j$-th dimension, 
$\mathrm{ub}_{j}$ and $\mathrm{lb}_{j}$ are the upper and lower bound of $j$-th dimension, respectively, $c_{1}, c_{3}$ are the random numbers,

$\mathrm{Lv}$ are the random values generated based on Levy Flight.

The pseudo code of iSSA is given in Fig. 5. The bold line indicates the proposed modification.

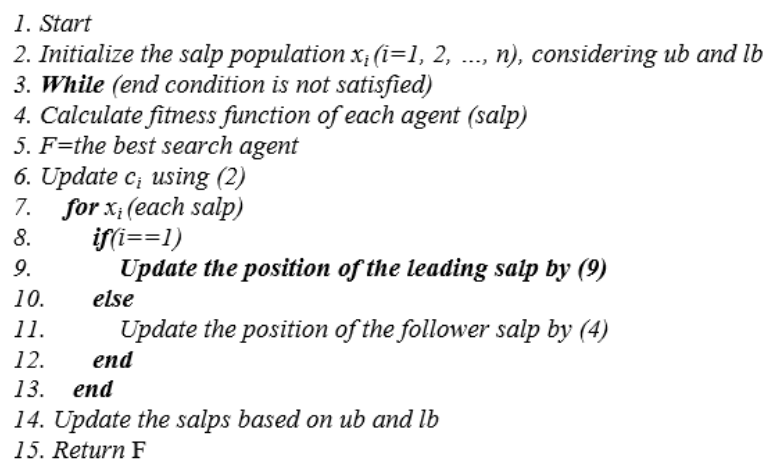

Fig. 5. Pseudo code of iSSA

\subsection{4. iSSA prediction model}

In this study, iSSA algorithm will be used as an estimator of parameters for dengue outbreak prediction. The inputs consist of humidity (hmdt), temperature (temp), rainfall (rain) and the dengue incidence number (dcases), in monthly frequency. Besides, the derivation of the stated inputs until month 6 are fed to the prediction model as well. Meanwhile, the output is the dengue cases from month 6 and onwards. The input and output configuration are defined as

$$
\begin{gathered}
\text { d_casesm6owd }=\left(x_{1} * \text { rain } \_\mathrm{ml}\right)+\ldots+\left(x_{6} * \text { temp } \_\mathrm{ml}\right)+\ldots+ \\
+\left(x_{11} * \text { hmdt_m1 }\right)+\ldots\left(x_{16} * \text { dcases_m1 }\right)+\ldots+x_{21},
\end{gathered}
$$

where:

d_casesm6owd are the dengue cases from month 6 and onwards; $x_{1}, x_{2}, \ldots, x_{21}$ is the coefficient to the respective employed variables; $\mathrm{m} 1, \mathrm{~m} 2, \ldots, \mathrm{m} 5$ - the month 1 to month 5; $x_{25}$ - constant.

\subsubsection{Evaluation}

To evaluate the efficiency of the proposed iSSA, two evaluation metrics were utilized namely Root Mean Square Error (RMSE). Both metrics are defined as

$$
\operatorname{RMSE}=\sqrt{\frac{\sum_{i=1}^{n}\left(y\left(x_{n}\right)-y_{n}\right)^{2}}{n}},
$$

where: 
$n=1,2, \ldots, N$,

$y_{n}$ are the target values,

$y\left(x_{n}\right)$ are the predict values,

$N$ is the total number of tests.

The lowest value of RMSE refers to the best method. Upon the prediction task, the normalized data using (8) (see Section 4.2) are denormalized back, based on the reverse of (8). This is important to ensure all evaluations are calculated based on original scale of the data.

\section{Results}

This section discusses the prediction performance recorded by iSSA and the identified algorithms namely SSA and ACO. In this study, the maximum iteration is set to 300 , while the optimized values for parameters of interest produced by iSSA, SSA and ACO are as tabulated in Table 5. Based on the Table 6, the RMSE produced by iSSA is 3.45, which is far smaller compared to SSA which recorded 28.46 of MSE. Meanwhile, ACO yielded 12.6 of RMSE, which is the largest compared to iSSA and SSA.

Table 5. Optimized parameters of interest for iSSA, SSA and ACO

\begin{tabular}{|c|c|c|c|}
\hline Parameters & iSSA & SSA & ACO \\
\hline$x_{1}$ & 0.0120 & 0.0070 & 0.4533 \\
\hline$x_{2}$ & 0.0105 & 0.0093 & 0.6392 \\
\hline$x_{3}$ & 0.0099 & 0.0156 & 0.4568 \\
\hline$x_{4}$ & 0.0295 & 0.0303 & 0.4571 \\
\hline$x_{5}$ & 0.0230 & 0.0069 & 0.4408 \\
\hline$x_{6}$ & 0.0157 & 0.0144 & 0.5190 \\
\hline$x_{7}$ & 0.0134 & 0.0103 & 0.6570 \\
\hline$x_{8}$ & 0.0075 & 0.0082 & 0.4052 \\
\hline$x_{9}$ & 0.0166 & 0.0156 & 0.5210 \\
\hline$x_{10}$ & 0.0073 & 0.0176 & 0.5470 \\
\hline$x_{11}$ & 0.0025 & 0.0091 & 0.4700 \\
\hline$x_{12}$ & 0.0030 & 0.0244 & 0.4767 \\
\hline$x_{13}$ & 0.0038 & 0.0207 & 0.4301 \\
\hline$x_{14}$ & 0.0271 & 0.0174 & 0.5556 \\
\hline$x_{15}$ & 0.0116 & 0.0163 & 0.6586 \\
\hline$x_{16}$ & 0.0038 & 0.01963 & 0.5010 \\
\hline$x_{17}$ & 0.0117 & 0.0165 & 0.5140 \\
\hline$x_{18}$ & 0.0119 & 0.0047 & 0.6028 \\
\hline$x_{19}$ & 0.0209 & 0.0147 & 0.3680 \\
\hline$x_{20}$ & 0.0040 & 0.0122 & 0.4127 \\
\hline$x_{21}$ & 0.0140 & 0.0104 & 0.5655 \\
\hline
\end{tabular}

Table 6. Comparison of iSSA vs SSA vs ACO for dengue outbreak prediction

\begin{tabular}{|c|c|c|c|}
\hline Algorithm & iSSA & SSA & ACO \\
\hline RMSE & $\mathbf{3 . 4 5}$ & 28.46 & 12.6 \\
\hline
\end{tabular}

The iSSA not only able to produce lowest RMSE of prediction rate, but the proposed iSSA is also competitive to converge faster (see Fig. 6) with lowest value, 
which is 6.5325 compared to SSA and ACO which recorded 7.8592 and 21.0380 respectively (see Fig. 7). The obtained results are further discussed in Section 6.

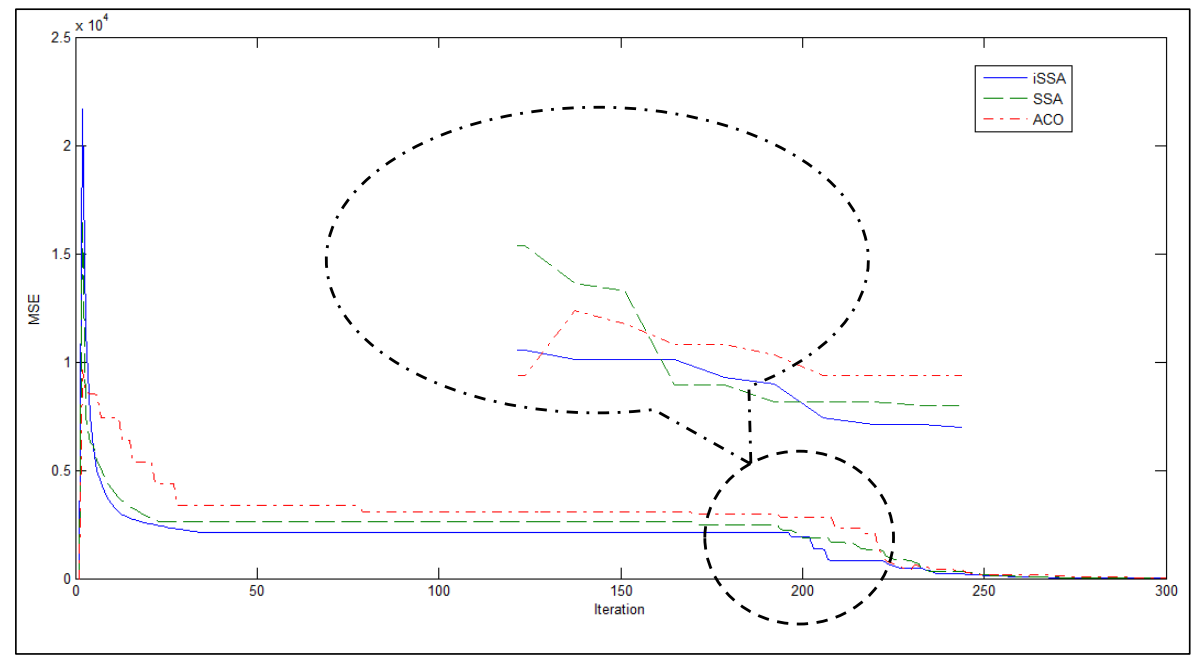

Fig. 6. Comparison of convergence value for iSSA vs SSA vs ACO

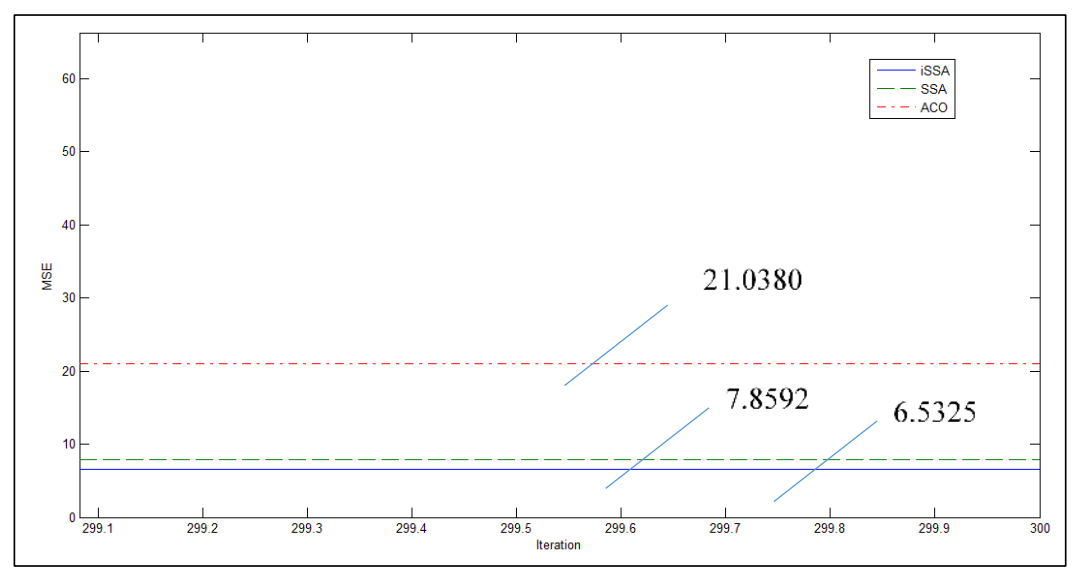

Fig. 7. Close-up of convergence value comparison: iSSA vs SSA vs ACO

\section{Discussion}

Based on the produced results by iSSA (see Section 5), the induced of Levy Flight to the conventional SSA has assisted the algorithm to have larger step length, which finally assist the algorithm from escaping the local optimum, hence optimal values for parameters of interest can be produced. With such feature, better prediction result can be obtained. The implementation of Levy Flight not only contribute in prediction task, but also significantly prove the convergence rate achieved by the iSSA, both in speed and value. With this capability, the algorithm possesses a great the feature of avoiding local optimum. 


\section{Conclusion}

In this study, an improved SSA based on Levy Flight is proposed, which is later known as iSSA, for monthly dengue outbreak prediction. The aim of inducing the Levy Flight to the SSA is to build up the exploration of SSA, hence global optimum can be obtained. The prediction model was fed with four variables which influence the occurrence of dengue cases namely, rainfall, temperature, humidity and the number of dengue incidence as well. For comparison purposes, the prediction performance of iSSA was compared against SSA and ACO, and guided by RMSE. Findings of the study suggested that the iSSA was not only able to produce lower RMSE, but also capable to converge faster with lower value. In the future, the study on the dengue outbreak prediction will be extended by referring to the methods applied in $[32,33,34,35]$.

Acknowledgements: The authors fully acknowledge the financial support from Fundamental Research Grant Scheme (FRGS), Ministry Of Higher Education (MOHE) (Grant No FRGS/1/2019/ICT02/UMP/02/4), and Faculty of Computing, Universiti Malaysia Pahang (https://fskkp.ump.edu.my/index.php/en/).

\section{References}

1. Z h a n g, P., H. N. W u, R. P. C h e n, T. H. T. Ch a n. Hybrid Meta-Heuristic and Machine Learning Algorithms for Tunneling-Induced Settlement Prediction: A Comparative Study. - Tunnelling and Underground Space Technology, Vol. 99, 2020, No 103383, pp. 1-13.

2. W u, L., G. H u a n g, J. F a n, X. M a, H. Z h o u, W. Z e n g. Hybrid Extreme Learning Machine with Meta-Heuristic Algorithms for Monthly Pan Evaporation Prediction. - Computer and Electronics in Agriculture, Vol. 168, 2020, No 105115, pp. 1-12.

3. D a s, S. R., D. M i s h r a, D. R o u t, M. R o u t. Stock Market Prediction Using Firefly Algorithm with Evolutionary Framework Optimized Feature Reduction for OSELM Method. - Expert Systems with Applications, Vol. 4, 2019, No 100016, pp. 1-24.

4. A $1 \mathrm{t}$ a n, A., S. K a r a s u, S. B e k i ro s. Digital Currency Forecasting with Chaotic Meta-Heuristic Bio-Inspired Signal Processing Techniques. - Chaos, Solitons \& Fractals, Vol. 126, 2019, No September 2019, pp. 325-336.

5. N a d e ri, M., E. K h a m e h c h i, B. K a r i m i. Novel Statistical Forecasting Models for Crude Oil Price, Gas Price, and Interest Rate Based on Meta-Heuristic Bat Algorithm. - Journal of Petroleum Science and Engineering, Vol. 172, 2019, No January 2019, pp. 13-22.

6. Mil a n, S. T., L. R a jabi o n, H. R a n jb ar, N. J. N a vi mi pour. Nature Inspired MetaHeuristic Algorithms for Solving the Load-Balancing Problem in Cloud Environments. Computers \& Operations Research, Vol. 110, 2019, No October 2019, pp. 159-187.

7. A $1 \mathrm{kh}$ an a k, E. N., S. P. Le e. A Hyper-Heuristic Cost Optimisation Approach for Scientific Workflow Scheduling in Cloud Computing. - Future Generation Computer Systems, Vol. 86, 2018, No September 2018, pp. 480-506.

8. R e d d y, S. S., P. R. B i j w e. - Efficiency Impruvements in Meta-Heuristic Algorithms to Solve the Optimal Power Flow Problem. - International Journal Electrical Power Energy Systems, Vol. 82, 2016, No November 2016, pp. 288-302.

9. S e g h i e r, M. E. A. B., B. Ke sht e g a r, K. F. Tee, T. Z a y ed, R. A b b a s si, N. T. Tru ng. Prediction of Maximum Pitting Corrosion Depth in Oil and Gas Pipelines. - Engineering Failure Analysis, Vol. 112, 2020, No 104505, pp. 1-14.

10. Ga mbhir, S., S. K. Malik, Y. Ku mar. PSO-ANN Based Diagnostic Model for the Early Detection of Dengue Disease. - New Horizons Translational Medicine, Vol. 4, 2017, No 1-4, pp. 1-8. 
11.A 1-Q a nes s, M. A. A., A. Ewees, H. Fan, M. A. E 1 A zi z. Optimization of Method for Forecasting Confirmed Cases of COVID-19 in China. - J. Clininal Med., Vol. 9, 2020, No 674, pp. 1-15.

12. S a p t a r i n i, N. G. A. P. H., R. Y. D i 11 a k, P. D. P a k a n. Dengue Haemorrhagic Fever Outbreak Prediction Using Elman Levenberg Neural Network and Genetic Algorithm. - In: Proc. of 2nd East Indonesia Conference on Computer and Information Technology (EIConCIT'18), 2018, pp. 188-191.

13. H u s i n, N. A., N. M u s t a p h a, M. N. S u 1 a i m a n, R. Y a a k o b. A Hybrid Model Using Genetic Algorithm and Neural Network for Predicting Dengue Outbreak. - In: Proc. of 4th Conference on Data Mining and Optimization (DMO), 2012, pp. 23-27.

14. Mustaffa, Z., M. H. Sula i man, M. F. M. Mohsin, Y. Yusof, F. Ernawan, K. A. M. R os li. An Application of Hybrid Swarm Intelligence Algorithms for Dengue Outbreak Prediction. - IEEE Jordan International Joint Conference on Electrical Engineering and Information Technology (JEEIT), 2019, pp. 731-735.

15. What is Dengue and How It Is Treated, 2017. who.int/news-room/q-a-detail/what-is-dengue-and-how-it-is-treated

16. Xu, Z., H. B a mbrick, L. Yakob, G. Devine, F. D. Frentiu, R. Marina, P. W. D h e w an t a ra, R. N u s a, R. T. S a s m o n o, W. H u. Using Dengue Epidemics and Local Weather in Bali, Indonesia to Predict Imported Dengue in Australia. - Environmental Research., Vol. 175, 2019, No 2019, pp. 213-220.

17. Cortes, F., C. M. T. Martelli, R. A. D. A. Ximenes, U. R. Montarroyos, J. B. S. J u n i o r, O. G. C r u z, N. A l e x a n d e r, W. V. D. S o u z a. Time Series Analysis of Dengue Surveillance Data in Two Brazillian Cities. - Acta Tropica, Vol. 182, 2018, No March 2018, pp. 190-197.

18. M irjalili, S., A. H. Gand o mi, S.Z.Mirjali li, S. S a re mi, H.F aris, S. M. Mirjalili. Salp Swarm Algorithm: A Bio-Inspired Optimizer for Engineering Design Problems. Advances in Engineering Software, Vol. 114, 2017, No December 2017, pp. 163-191.

19. K a n s a 1, V., J. S. D h 111 o n. Emended Salp Swarm Algorithm for Multiobjective Electric Power Dispatch Problem. - Applied Soft Computing, Vol. 90, 2020, No 106172, pp. 1-26.

20. N e g g a z, N., A. A. E w e e s, M. A. E 1 a z i z, M. M a f a r j a. Boosting Salp Swarm Algorithm by Sine Cosine Algorithm and Disrupt Operator for Feature Selection. - Expert Systems with Applications, Vol. 145, 2020, No 113103, pp. 1-20.

21. Q a i s, M. H., H. M. H a s a n i en, S. A $1 \mathrm{~g}$ h u w a in e m. Enhanced Salp Swarm Algorithm: Application to Variable Speed Wind Generators. - Engineering Applications of Artificial Intelligence, Vol. 80, 2019, No April 2019, pp. 82-96.

22. Tu b i sh a t, M., N. Idri s, L. Sh u ib, M. A. M. A b u sh ari a h, S. M ir jalili. Improved Salp Swarm Algorithm Based on Opposition Based Learning and Novel Local Search Algorithm for Feature Selection. - Expert Systems with Applications, Vol. 145, 2020, No 113122, pp. 1-10.

23. G h o 1 a m i, K., M. H. P a r v a n e h. A Mutated Salp Swarm Algorithm for Optimum Allocation of Active and Reactive Power Sources in Radial Distribution Systems. - Applied Soft Computing, Vol. 85, 2019, No 105833, pp. 1-14.

24. Ateya, A. A., A. Muthanna, A. Vybornova, A. D. Algarni, A. Abuarqoub, Y. K o u c h e r y a v y, A. K o u c h e r y a v y. Chaotic Salp Swarm Algorithm for SDN MultiController Networks. - Engineering Science and Technology an International Journal, Vol. 22, 2019, No 4, pp. 1001-1012.

25. Le v y, P. Theorie de l'Addition des Veriables Aleatoires. Paris, France, Gauthier-Villars, 1937. 26. Salp. https://en.wikipedia.org/wiki/Salp

27. L i u, M., X. Y a o, Y. L i. Hybrid Whale Optimization Algorithm Enhanced with Lévy Flight and Differential Evolution for Job Shop Scheduling Problems. - Applied Soft Computing, Vol. 87, 2020, No105954, pp. 1-16.

28. E m a r y, E., H. M. Z a w b a a, M. S h a r a w i. Impact of Lèvy Flight on Modern Meta-Heuristic Optimizers. - Applied Soft Computing, Vol. 75, 2019, No February 2019, pp. 775-789. 
29. Che g in i, S. N., A. B a g h e ri, F. N a j a fi. PSOSCALF: A New Hybrid PSO Based on Sine Cosine Algorithm and Levy Flight for Solving Optimization Problems. - Applied Soft Computing, Vol. 73, 2018, No December 2018, pp. 697-726.

30. Zh ang, Y., Z. J i n, X. Z h a o, Q. Y a n g. Backtracking Search Algorithm with Lévy Flight for Estimating Parameters of Photovoltaic Models. - Energy Conversion and Management, Vol. 208, 2020, No 112615, pp. 1-15.

31. No Title.

https://github.com/alramadona/yews4denv/tree/master/data

32. Terziyska, M., Y. Todorov, D. Miteva, M. Doneva, S. Dyankova, P. M e t o d i e v a, I. N a c h e v a. Bayesian Regularized Neural Network for Prediction of the Dose in Gamma Irradiated Milk Products. - Cybernetics and Information Technologies, Vol. 20, 2020, No 2, pp. 141-151.

33. T o s h e v, A. Particle Swarm Oprimization and Tabu Search Hybrid Algorithm for Flexible Job Shop Scheduling Problem - Analysis of Test Result. - Cybernetics and Information Technologies, Vol. 19, 2019, No 4, pp. 26-44.

34. Yusob, B., Z. Mustaffa, J. Sulaiman. Anomaly Detection in Time Series Data Using Spiking Neural Network. - Journal of Computational and Theoretical Nanoscience, Vol. 24, 2018, No 10, pp. 7572-7576.

35. Fird a u s, A., N. B. A n u ar, M. F. A. R a z a k, A. K. S a n g a i a h. Bio Inspired Computational Paradigm for Feature Investigation and Malware Detection: Interactive Analytics. Multimedia Tools and Applications, Vol. 77, 2018, No 2018, pp. 17519-17555.

Received: 24.04.2020; Second Version: 24.07.2020; Accepted: 25.08.2020 